\title{
Percutaneous Nephrolithotomy Can Reduce the Incidence of Sepsis Compared with Flexible Ureteroscopy in Treating Solitary Proximal Ureteral Stone Patients with Positive Urine Culture
}

\author{
Qi-Dong Xia $(\mathbb{D}$, Yu-Feng Wang, Chen-Qian Liu, Jin-Zhou Xu, Jian-Xuan Sun, Yang Xun, \\ Cong Li, Jia Hu, Jun-Lin Lu $\mathbb{D}$, and Shao-Gang Wang $\mathbb{D}$
}

Department of Urology, Tongji Hospital, Tongji Medical College, Huazhong University of Science and Technology, Wuhan, China

Correspondence should be addressed to Jun-Lin Lu; lujunlintjm@hust.edu.cn and Shao-Gang Wang; sgwangtjm@163.com

Received 4 March 2021; Revised 25 March 2021; Accepted 29 March 2021; Published 13 April 2021

Academic Editor: Manash K. Paul

Copyright (C) 2021 Qi-Dong Xia et al. This is an open access article distributed under the Creative Commons Attribution License, which permits unrestricted use, distribution, and reproduction in any medium, provided the original work is properly cited.

\begin{abstract}
Background. Sepsis is a potentially lethal complication for both flexible ureteroscopy (fURS) and percutaneous nephrolithotomy (PCNL). This study is aimed at comparing the sepsis rate after fURS and PCNL and the risk factors for sepsis in patients with solitary proximal ureteral stone. Methods. We reviewed the data of patients with calculi between $10 \mathrm{~mm}$ to $20 \mathrm{~mm}$ who underwent fURS or PCNL surgery from Tongji Hospital's database. A total of 910 patients were eligible with 412 fURS cases and 498 PCNL cases. We used univariate analysis and multivariate logistic regression analysis to identify the risk factors for sepsis. Subgroup analysis was performed using logistic regression analysis. Results. In the cohort, 27 (6.6\%) and 19 (3.8\%) patients developed sepsis after fURS and PCNL, respectively. Multivariate analysis shows that the risk factors for sepsis are fURS $(\mathrm{OR}=3.160, P=0.004)$, serum $\mathrm{WBC} \geq 10,000$ cells $/ \mu \mathrm{L}(\mathrm{OR}=3.490, P=0.008)$, albumin - globulin ratio $<1.2(\mathrm{OR}=2.192, P=$ $0.029)$, positive urine culture $(\mathrm{OR}=6.145, P<0.001)$, and prolonged operation time $(\mathrm{OR}=1.010, P=0.046)$. Subgroup analysis was conducted using potential risk factors: stone size, serum WBC, urine culture, and albumin-globulin ratio (AGR). In subgroup of positive urine culture, patients were more likely to develop sepsis after fURS than PCNL. Conclusions. PCNL may be a better choice than fURS to reduce postoperative sepsis, especially for patients with positive urine culture.
\end{abstract}

\section{Introduction}

Urolithiasis is one of the most common diseases in the urinary system. It affects patients globally because of its high incidence rate that is $7-13 \%$ in North America, $5-9 \%$ in Europe, and $1-5 \%$ in Asia [1-3]. Surgery is the main treatment for urolithiasis: extracorporeal shock wave lithotripsy, endoscopic surgery, or laparoscopic surgery. Among them, percutaneous nephrolithotomy (PCNL) and flexible ureteroscopy (fURS) are both recommended to remove $10-20 \mathrm{~mm}$ ureteral stone [4].

Sepsis is one of the most intractable surgery complications, which leads to a longer length of stay and even lethal sepsis shock in some cases [5]. According to previous studies, postoperative sepsis is the primary complication with an incidence of $0.3-7.4 \%$ in fURS and $0.9-5.9 \%$ in PCNL [6-8]. It seems that different surgical procedures may lead to different incidence rate of sepsis. Thus, urologists make efforts to discover risk factors or preoperative predicting factors for postoperative sepsis. There have been several preoperative features identified as risk factors such as positive urine culture, female sex, and diabetes [9]. However, though the preoperative risk factor has been identified, it is hardly helpful for clinicians to make a clinical decision about which surgical procedure to choose, PCNL or fURS. Thus, we aim to compare the occurrence of postoperative sepsis between fURS and PCNL and analyze the difference of risk factors.

Besides, PCNL is usually utilized to treat larger stone than fURS $[10,11]$. Considering the heterogeneity of the stone size between patients may influence the sepsis incidence, we conducted a retrospective clinical data collection of patients with solitary proximal ureteral stone with stone size between 10 and $20 \mathrm{~mm}$. We performed univariate and multivariate analyses to discover the risk factors for postoperative sepsis in our 


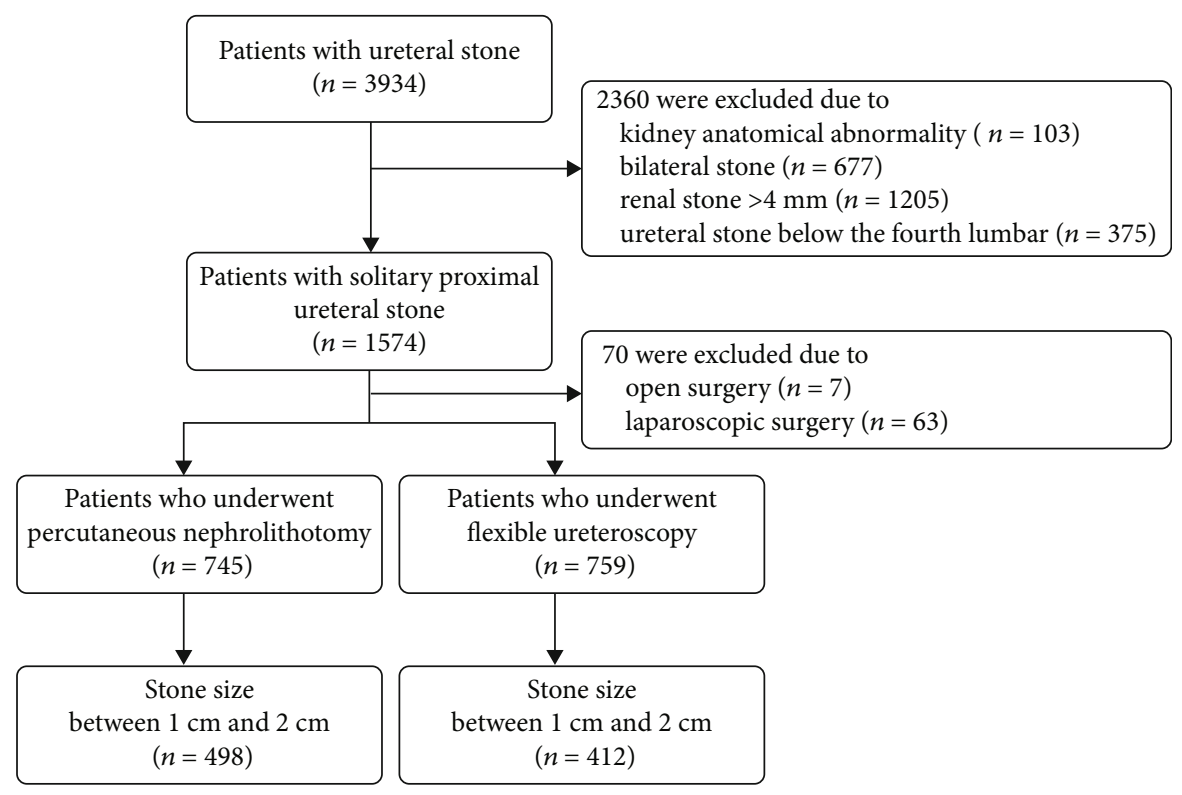

Figure 1: The screening flow chart.

cohort. Our works may provide evidence for clinicians to make surgical choice.

\section{Materials and Methods}

The research was approved by the Ethics Committee of Tongji Medical College (2019S1035). The retrospective study included patients from January 2012 to December 2018. The inclusion criteria were (1) unilateral, solitary, and proximal ureteral stones; (2) PCNL or fURS to treat urolithiasis; (3) stone size ranging from $10 \mathrm{~mm}$ to $20 \mathrm{~mm}$; (4) patient age $\geq$ 18 years. The exclusion criteria were anatomical abnormality: solitary kidney, horseshoe kidney, transplant kidney, and kidney duplication.

The primary outcome was postoperative sepsis. According to the 2001 International Sepsis Definitions Conference, postoperative sepsis was defined as the concurrence of infection and at least two of the following criteria with 48 hours of surgery: (1) heart rate $>90 /$ minute, (2) respiratory rate $>20$ /minute, (3) body temperature $>38^{\circ} \mathrm{C}$, and leukocyte count $<4,000$ cells $/ \mu \mathrm{L}$ or $>12,000$ cells $/ \mu \mathrm{L}$.

The patient data was retrospectively collected from the hospital's database. Preoperative factors were recorded such as patient age, sex, body mass index (BMI), comorbidities (diabetes, coronary heart disease, paraplegia, and hypertension), stone size and laterality, presence of hydronephrosis and indwelling stent, hematological tests (serum white blood cell [WBC], neutrophil, and lymphocyte), biochemical tests (creatinine, cholesterol, albumin, and globulin), urine tests (urine WBC and urine nitrite), urine culture, and American Society of Anesthesiologists (ASA) score. Size of ureteral access sheath and flexible ureteroscope for fURS and size of sheath and nephroscope for PCNL were also recorded. Operation time was documented from the commencement of operation to the end of anesthesia. The laboratory tests were routinely performed and obtained for all patients. Patients who have infectious indicators (fever, high serum WBC proportion, or positive urine culture) received at least a full antibiotic regimen for seven days until the tests turned negative. Otherwise, one dose of antibiotics was applied for prophylactic purpose.

Statistical analysis was conducted using Statistical Product and Service Solutions (SPSS) version 24.0. The Student's $t$-test was used to compare continuous variables (expressed by mean \pm standard deviation) with a normal distribution. Continuous variables with a skewed distribution were showed as median (interquartile range [IQR]) and compared by the Mann-Whitney $U$ test. The chi-square test or Fisher's exact test was utilized to detect the difference between groups with categorical variables (expressed by proportions). The logistic regression method was used to identify the risk factors of sepsis. The difference was considered statistically significant when $P$ value $<0.05$.

\section{Results}

After reviewing 3934 patients with ureteral stone, 2360 were excluded primarily due to kidney anatomical abnormality $(n=103)$, bilateral stone $(n=677)$, renal stone $>4 \mathrm{~mm}$ $(n=1205)$, and ureteral stone below the fourth lumbar $(n=375)$. Finally, a total of 910 patients with $10-20 \mathrm{~mm}$ solitary proximal ureteral stone were eligible for analysis (Figure 1). Among them, 412 patients underwent fURS, whereas 498 patients received PCNL.

The detailed basic information of the eligible patients is shown in Table 1. Patients who received PCNL had higher rate of hydronephrosis, larger stone size, and longer operation time. The rough sepsis rate of fURS (6.6\%) is higher than that of PCNL (3.8\%). But the difference is not significant $(P=0.061)$. The multivariate analysis indicates that five variables are independent risk factors of sepsis (Table 2): fURS $(\mathrm{OR}=3.160, \quad P=0.004), \quad$ serum $\mathrm{WBC} \geq 10,000 \quad$ cells $/ \mu \mathrm{L}$ 
TABLE 1: Basic characteristics of including patients.

\begin{tabular}{|c|c|c|c|c|}
\hline Variables & All patients $(n=910)$ & fURS $(n=412)$ & PCNL $(n=498)$ & $P$ value \\
\hline Age (years) & $50.0 \pm 12.4$ & $49.1 \pm 13.2$ & $50.6 \pm 11.6$ & 0.074 \\
\hline Male, $n(\%)$ & $588(64.6)$ & $271(65.8)$ & $317(63.7)$ & 0.505 \\
\hline BMI $\left(\mathrm{kg} / \mathrm{m}^{2}\right)$ & $24.0 \pm 3.1$ & $24.2 \pm 3.3$ & $23.9 \pm 3.0$ & 0.163 \\
\hline \multicolumn{5}{|l|}{ Preoperative urological condition, $n(\%)$} \\
\hline Hydronephrosis & $131(14.4)$ & $34(8.3)$ & $97(19.5)$ & $<0.001$ \\
\hline Indwelling stent & $63(6.9)$ & $29(7.0)$ & $34(6.8)$ & 0.900 \\
\hline \multicolumn{5}{|l|}{ Stone characteristics } \\
\hline Stone size $(\mathrm{mm})$ & $13.3 \pm 3.2$ & $12.4 \pm 2.8$ & $14.1 \pm 3.2$ & $<0.001$ \\
\hline Left side, $n(\%)$ & $476(52.3)$ & $208(50.5)$ & $268(53.8)$ & 0.317 \\
\hline \multicolumn{5}{|l|}{ Comorbidities, $n$ (\%) } \\
\hline Diabetes & $74(8.1)$ & $39(9.5)$ & $35(7.0)$ & 0.180 \\
\hline Coronary heart disease & $15(1.6)$ & $6(1.5)$ & $9(1.8)$ & 0.679 \\
\hline Hypertension & $210(23.1)$ & $90(21.8)$ & $120(24.1)$ & 0.422 \\
\hline \multicolumn{5}{|l|}{ Urine test } \\
\hline Urine WBC, median (IQR) (cells/hpf) & $52.0(19.6-163.7)$ & $46.0(17.1-127.4)$ & $59.0(21.3-193.4)$ & 0.024 \\
\hline Positive urine nitrite, $n(\%)$ & $55(6.0)$ & $23(5.6)$ & $32(6.4)$ & 0.595 \\
\hline Positive urine culture, $n(\%)$ & $100(11.0)$ & $38(9.2)$ & $62(12.4)$ & 0.121 \\
\hline \multicolumn{5}{|l|}{ Hematological test $\left(10^{9} \mathrm{cells} / \mathrm{L}\right)$} \\
\hline Serum WBC & $6.8 \pm 2.8$ & $6.9 \pm 3.2$ & $6.6 \pm 2.5$ & 0.164 \\
\hline Neutrophil & $4.1 \pm 2.7$ & $4.3 \pm 3.0$ & $4.0 \pm 2.4$ & 0.169 \\
\hline Lymphocyte & $1.9 \pm 0.6$ & $1.9 \pm 0.6$ & $1.9 \pm 0.6$ & 0.943 \\
\hline \multicolumn{5}{|l|}{ Biochemical test } \\
\hline Albumin $(\mathrm{g} / \mathrm{L})$ & $39.5 \pm 4.2$ & $39.8 \pm 4.3$ & $39.3 \pm 4.3$ & 0.662 \\
\hline Globulin (g/L) & $29.2 \pm 4.6$ & $29.2 \pm 4.4$ & $29.2 \pm 4.8$ & 0.061 \\
\hline AGR & $1.39 \pm 0.28$ & $1.39 \pm 0.27$ & $1.39 \pm 0.29$ & 0.051 \\
\hline Creatinine, median $(\mathrm{IQR})(\mu \mathrm{mol} / \mathrm{L})$ & $85.0(69.0-108.0)$ & $83.0(68.0-108.0)$ & $86.0(70.0-109.0)$ & 0.680 \\
\hline Cholesterol $(\mathrm{mmol} / \mathrm{L})$ & $4.1 \pm 0.9$ & $4.1 \pm 0.9$ & $4.1 \pm 0.9$ & 0.848 \\
\hline$A S A, n(\%)$ & & & & 0.150 \\
\hline I & $378(41.5)$ & $157(38.1)$ & $221(44.4)$ & \\
\hline II & $510(56.0)$ & $243(59.0)$ & $267(53.6)$ & \\
\hline III & $21(2.3)$ & $12(2.9)$ & $9(1.8)$ & \\
\hline IV & $1(0.1)$ & $0(0.0)$ & $1(0.2)$ & \\
\hline Operation time, median (IQR) (min) & $85.0(66.0-110.0)$ & $72.5(58.0-89.0)$ & $98.0(78.0-120.0)$ & $<0.001$ \\
\hline Postoperative sepsis, $n(\%)$ & $46(5.1)$ & $27(6.6)$ & $19(3.8)$ & 0.061 \\
\hline
\end{tabular}

(OR $=3.490, \quad P=0.008), \quad$ albumin - globulin ratio $<1.2$ $(\mathrm{OR}=2.192, P=0.029)$, positive urine culture $(\mathrm{OR}=6.145$, $P<0.001)$, and prolonged operation time $(\mathrm{OR}=1.010$, $P=0.046)$. Sex $(P=0.354)$ and stone size $(P=0.716)$ are not considered as independent risk factors.

Urologists are more likely to choose PCNL to treat larger stones. We divided patients into a larger stone size group (15$20 \mathrm{~mm})$ and a smaller stone size group (10-15 mm) to evaluate the sepsis rate between fURS and PCNL (Figure 2). Both groups are not of statistical difference: $P=0.160$ for smaller stone size group and $P=0.205$ for larger stone size group. Subgroup analysis using sepsis risk factors is also performed: serum WBC, urine culture, and albumin-globulin ratio (AGR). In the positive urine culture group, patients who receive fURS have 5.71 times the risk of developing sepsis than patients who receive PCNL $(P<0.001)$.

\section{Discussion}

Sepsis is one of the most severe complications in patients who underwent lithotomy, which may both occur after PCNL or fURS $[12,13]$. It has been reported that the occurrence rate of sepsis post-fURS or post-PCNL was different. According to previous research, the sepsis rate after fURS reaches 
TABLE 2: Multivariate analysis for the risk factors of sepsis.

\begin{tabular}{|c|c|c|c|c|}
\hline Variables & $\mathrm{B}$ & OR & $95 \% \mathrm{CI}$ & $P$ value \\
\hline Surgery (ref. PCNL) & 1.151 & 3.160 & (1.459 to 6.842$)$ & 0.004 \\
\hline Sex (ref. male) & 0.324 & 1.383 & (0.697 to 2.747$)$ & 0.354 \\
\hline Age (years) & 0.019 & 1.019 & (0.990 to 1.048$)$ & 0.200 \\
\hline BMI $\left(\mathrm{kg} / \mathrm{m}^{2}\right)$ & 0.013 & 1.014 & (0.910 to 1.129$)$ & 0.807 \\
\hline Stone size $(\mathrm{mm})$ & 0.020 & 1.021 & (0.915 to 1.139$)$ & 0.716 \\
\hline Indwelling stent & -0.038 & 0.962 & $(0.338$ to 2.739$)$ & 0.943 \\
\hline Diabetes & -0.431 & 0.650 & $(0.181$ to 2.334$)$ & 0.509 \\
\hline Hydronephrosis & -0.519 & 0.595 & (0.168 to 2.107$)$ & 0.421 \\
\hline Serum $W B C \geq 10,000$ cells $/ \mu \mathrm{L}$ & 1.250 & 3.490 & (1.391 to 8.758$)$ & 0.008 \\
\hline $\mathrm{AGR}<1.2$ & 0.785 & 2.192 & (1.082 to 4.442$)$ & 0.029 \\
\hline Positive urine culture & 1.816 & 6.145 & (2.541 to 14.859$)$ & $<0.001$ \\
\hline Urine WBC $\geq 50$ cells/hpf & 0.185 & 1.203 & (0.531 to 2.726$)$ & 0.659 \\
\hline Positive urine nitrite & 0.676 & 1.967 & $(0.750$ to 5.157$)$ & 0.169 \\
\hline Operation time (min) & 0.010 & 1.010 & (1.000 to 1.020$)$ & 0.046 \\
\hline
\end{tabular}

Abbreviations: PCNL: percutaneous nephrolithotomy; BMI: body mass index; WBC: white blood cell; AGR: albumin globulin ratio.

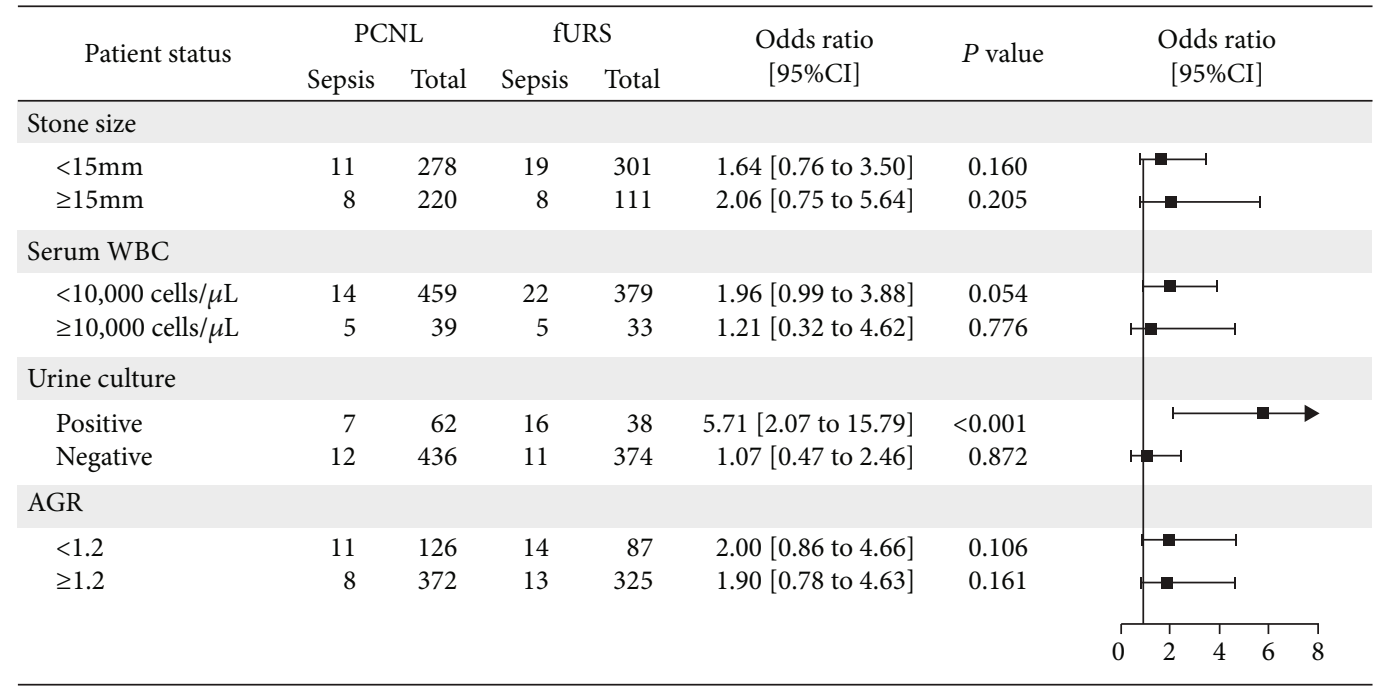

FIGURE 2: Subgroup analysis to compare sepsis rate between PCNL and fURS.

$0.3 \%-7.4 \%[6,7]$, and the sepsis rate after PCNL reaches 0.9\%-5.9\% [8]. In our study, sepsis after PCNL was $3.8 \%$ (19/498) and 6.6\% (27/412) after fURS, which shows a consistency with previous studies. Based on the multivariate logistic analysis, we find that surgical option, positive urine culture, serum WBC, and operation time are independent predictors of postoperative sepsis. Patients with positive urine culture are more likely to suffer sepsis after fUSR than PCNL. However, no significant difference in sepsis rate is indicated between PCNL and fURS when urine culture is negative.

We previously report that positive urine culture is an independent predictor for post-fURS sepsis and assemble a nomogram to predict the occurrence of post-fURS sepsis [14]. Uchinda [15] et al. and Blackmur et al. [7] explored the role of bladder urine culture in infectious complications that it is an independent risk factor increasing 3.53 to 4.88 times the risk of infectious complications. It may be because the high intrarenal pressure during the fURS promotes local pathogens and toxins into blood circulation. AGR usually plays a role as a predictor of cancer progression or cancerspecific survival because it reflects patients' nutrition, inflammation, and immunity [16]. We also find it as a predictor of sepsis after endourological stone surgery [14, 17]. In this study, both low AGR and high AGR groups, PCNL, and fURS have similar sepsis rates. From the aspect of reducing operative sepsis, the level of AGR may have little influence on surgical option.

Kreydin and Eisner have systemically summarized the risk factors for sepsis after PCNL [18], and the pre-PCNL factors included positive urine culture, female, nephrostomy, urinary diversion, stone size, hydronephrosis, diabetes, and 
complicated calculi. Meanwhile, the local urinary system condition is considered the most critical factor related to infectious complications. Besides, more novel predictors for post-PCNL sepsis are identified by researchers. For example, C-reactive protein, albumin, and procalcitonin are considered predictors reflecting the systematic condition of patients $[8,19]$.

Positive urine culture is a predictor of postoperative sepsis for both fURS and PCNL. The evidence reveals that urine culture can be an essential reference factor for surgical choice to reduce the incidence of sepsis. In patients with $10-20 \mathrm{~mm}$ ureteral stone, we find that PCNL is better than fURS when patients have a positive urine culture. Our works can optimize the surgery strategy for patients with a high risk of infection $[20,21]$.

The main limitation of the study is the single-center retrospective nature, which may cause selection bias. We include a relatively large number of patients to stabilize the results. Subgroup analysis is also performed to compare sepsis incidence in patients with different conditions. Further prospective and multicenter studies are needed.

\section{Conclusions}

In summary, we find that PCNL might be a better choice than fURS to reduce postoperative sepsis, especially when patients have a positive urine culture.

\section{Abbreviations}

fURS: Flexible ureteroscopy

PCNL: Percutaneous nephrolithotomy

BMI: Body mass index

WBC: White blood cell

AGR: Albumin-globulin ratio

ASA: American Society of Anesthesiologists

SPSS: Statistical Product and Service Solutions

IQR: Interquartile range.

\section{Data Availability}

All data that supports the findings of this study is available from the corresponding author upon reasonable request.

\section{Ethical Approval}

The authors are accountable for all aspects of the work in ensuring that questions related to the accuracy or integrity of any part of the work are appropriately investigated and resolved. The authors are accountable for all aspects of the work in ensuring that questions related to the accuracy or integrity of any part of the work are appropriately investigated and resolved. The study was conducted in accordance with the Declaration of Helsinki (as revised in 2013). The study was approved by the Ethics Committee of Tongji Medical College, Huazhong University of Science and Technology (IORG No: IORG0003571).

\section{Consent}

Individual consent for this retrospective analysis was waived.

\section{Conflicts of Interest}

The authors declare that they have no conflicts of interest.

\section{Authors' Contributions}

(I) Conception and design was carried out by XQD, WYF, LJL, and WSG. (II) LC, HJ, and WSG contributed to the administrative support. (III) Provision of study materials or patients was done by LC and XY. (IV) Collection and assembly of data was performed by LJL and XQD. (V) LJL, WYF, and XQD contributed to the data analysis and interpretation. (VI) Manuscript writing and final approval of manuscript were done by all authors. Qi-Dong Xia and Yu-Feng Wang share co-first authorship.

\section{Acknowledgments}

This research is supported by grants from the National key research and development program (2017YFB1302804).

\section{References}

[1] M. J. Favus, "Epidemiology of Nephrolithiasis," in Primer on the Metabolic Bone Diseases and Disorders of Mineral Metabolism: Eighth Edition, pp. 856-859, Wiley Blackwell, 2013.

[2] I. Pinduli, R. Spivacow, E. Del Valle et al., "Prevalence of urolithiasis in the autonomous city of Buenos Aires, Argentina," Urological Research, vol. 34, no. 1, pp. 8-11, 2006.

[3] M. Medina-Escobedo, M. Zaidi, E. Real-de León, and S. Orozco-Rivadeneyra, "Urolithiasis prevalence and risk factors in Yucatan, Mexico," Salud Pública de México, vol. 44, no. 6, pp. 541-545, 2002.

[4] C. C. Wen and S. Y. Nakada, "Treatment selection and outcomes: renal calculi," Urologic Clinics of North America, vol. 34, no. 3, pp. 409-419, 2007.

[5] A. Heinze, A. S. Gozen, and J. Rassweiler, "Tract sizes in percutaneous nephrolithotomy: does miniaturization improve outcome?," Current Opinion in Urology, vol. 29, no. 2, pp. 118123, 2019.

[6] B. K. Somani, G. Giusti, Y. Sun et al., "Complications associated with ureterorenoscopy (URS) related to treatment of urolithiasis: the Clinical Research Office of Endourological Society URS Global study," World Journal of Urology, vol. 35, no. 4, pp. 675-681, 2017.

[7] J. P. Blackmur, N. U. Maitra, R. R. Marri, F. Housami, M. Malki, and C. Mcilhenny, "Analysis of factors' association with risk of postoperative urosepsis in patients undergoing ureteroscopy for treatment of stone disease," Journal of Endourology, vol. 30, no. 9, pp. 963-969, 2016.

[8] M. Rivera, B. Viers, P. Cockerill, D. Agarwal, R. Mehta, and A. Krambeck, "Pre- and postoperative predictors of infection-related complications in patients undergoing percutaneous nephrolithotomy," Journal of Endourology, vol. 30, no. 9, pp. 982-986, 2016.

[9] Y. Xun, Y. Yang, X. Yu, C. Li, J. Lu, and S. Wang, “A preoperative nomogram for sepsis in percutaneous nephrolithotomy 
treating solitary, unilateral and proximal ureteral stones," PeerJ, vol. 6, article e9435, 2020.

[10] C. Türk, A. Petř́k, K. Sarica et al., "EAU guidelines on interventional treatment for urolithiasis," European Urology, vol. 69, no. 3, pp. 475-482, 2016.

[11] G. M. Preminger, D. G. Assimos, J. E. Lingeman, S. Y. Nakada, M. S. Pearle, and J. S. Wolf, "Chapter 1: AUA guideline on management of staghorn calculi: diagnosis and treatment recommendations," The Journal of Urology, vol. 173, no. 6, pp. 1991-2000, 2005.

[12] T. Tokas, T. R. W. Herrmann, A. Skolarikos, and U. Nagele, "Pressure matters: intrarenal pressures during normal and pathological conditions, and impact of increased values to renal physiology," World Journal of Urology, vol. 37, no. 1, pp. 125-131, 2019.

[13] P. J. S. Osther, "Risks of flexible ureterorenoscopy: pathophysiology and prevention," Urolithiasis, vol. 46, no. 1, pp. 59-67, 2018.

[14] J. Lu, Y. Xun, X. Yu et al., “Albumin-globulin ratio: a novel predictor of sepsis after flexible ureteroscopy in patients with solitary proximal ureteral stones," Translational Andrology and Urology, vol. 9, no. 5, pp. 1980-1989, 2020.

[15] Y. Uchida, R. Takazawa, S. Kitayama, and T. Tsujii, "Predictive risk factors for systemic inflammatory response syndrome following ureteroscopic laser lithotripsy," Urolithiasis, vol. 46, no. 4, pp. 375-381, 2018.

[16] G. Y. Lv, L. An, X. D. Sun, Y. L. Hu, and D. W. Sun, "Pretreatment albumin to globulin ratio can serve as a prognostic marker in human cancers: a meta-analysis," Clinica Chimica Acta, vol. 476, pp. 81-91, 2018.

[17] T. Yang, S. Liu, J. Hu, L. Wang, and H. Jiang, “The evaluation of risk factors for postoperative infectious complications after percutaneous nephrolithotomy," BioMed Research International, vol. 2017, Article ID 4832051, 7 pages, 2017.

[18] E. I. Kreydin and B. H. Eisner, "Risk factors for sepsis after percutaneous renal stone surgery," Nature Reviews Urology, vol. 10, no. 10, pp. 598-605, 2013.

[19] C. G. Xu and Y. L. Guo, "Diagnostic and prognostic values of BMPER in patients with urosepsis following ureteroscopic lithotripsy," BioMed Research International, vol. 2019, Article ID 8078139, 9 pages, 2019.

[20] S. Chugh, A. Pietropaolo, E. Montanari, K. Sarica, and B. K. Somani, "Predictors of urinary infections and urosepsis after ureteroscopy for stone disease: a systematic review from EAU section of urolithiasis (EULIS)," Current Urology Reports, vol. 21, no. 4, p. 16, 2020.

[21] D. Chen, C. Jiang, X. Liang et al., "Early and rapid prediction of postoperative infections following percutaneous nephrolithotomy in patients with complex kidney stones," BJU International, vol. 123, no. 6, pp. 1041-1047, 2019. 